\title{
Citoyens sans frontières
}

\author{
Danilo Santos de Miranda
}

\section{Résumé}

Le Brésil est devenu actuellement un pays d'accueil pour des milliers de latino-américains et d'africains, surtout ceux de langue portugaise. L'article relève du travail des organisations de la société civile envers ces refugiés, en particulier l'effort du SESC (Service Social du Commerce), une institution sui generis d'action socioculturelle en Amérique Latine.

\section{Abstract \\ Brazil has become a host country for thousands of Latin- American and African people, especially those who speak Portuguese. This article discusses the activities of non- governmental organizations working with these refugees; more specifically, the efforts of SESC (Social Service of Trade), a sui generis institution engaged in socio-cultural activities in Latin America.}

$\mathrm{V}$ ictimes de conflits ethniques, de guerres, de persécutions politiques ou religieuses, ou simplement miséreux sociaux et économiques, les réfugiés affrontent fréquemment un nouvel obstacle dans le pays qui leur donne asile : celui qui rend difficile leur reconnaissance comme citoyen et par conséquent, comme personne ayant des droits sociaux et économiques, indispensables au rétablissement d'une vie normale et socialement digne.

Sebastião Salgado, le célèbre photographe brésilien dont l'œuvre montre la réalité de ces abandonnés, affirme dans Êxodos:

Les réfugies et les personnes déplacées se distinguent des immigrants car ils ne rêvent pas d'une vie différente. En général, ce sont des personnes communes, des « civils innocents, » qui mènent leur vie d'agriculteurs, d'étudiants ou de femmes au foyer jusqu'au moment où leurs destins s'associent violemment à la répression ou la guerre. D'un seul coup, ayant perdu leur maison, leur travail et parfois des êtres chers, ils sont dépouillés même de leur propre identité. Ils deviennent des gens en fuite, des visages sur des journaux télévisés ou des photographies, des chiffres dans des camps de réfugiés, des queues pour distribution d'aliments. Contrat cruel qu'est le leur : en échange de leur survie ils abdiquent de leur dignité. Ils ont rarement, en outre, les conditions de retrouver une vie normale, tout du moins, comme celle qu'ils avaient avant. Certains deviennent des réfugiés permanents, habitants des camps de réfugiés, comme les Palestiniens au Liban. Il est bien courant que les réfugiés n'ont pas de voix au chapitre des conflits politiques, ethniques et religieux qui dégénèrent en atrocités. Comment consoler ces gens, qui on assisté à ce que l'humanité a de pire?

Actuellement, le Brésil est un choix pour beaucoup de personnes en quête de refuge, venant d'Amérique du Sud (notamment Bolivie, Pérou et Colombie) et d'Afrique (Congo, Angola et Mozambique). Ils sont environ 3 200, parmi lesquels 1600 se trouvent à São Paulo. Ce contingent a augmenté, surtout à partir de 1997, lorsque, finalement, le dispositif international d'accueil, crée après la seconde guerre, a été réglementé par le gouvernement fédéral. Selon la loi 9.474, « a le droit d'habiter le Brésil tout citoyen dont le pays se trouve en situation de menace généralisée aux droits humains. " Et même si le nombre de demandes d'asile au gouvernement brésilien soit bien inférieur à celui aux pays développés, comme la France ou le Canada, la quantité de requêtes refusées par le gouvernement brésilien n'est pas très différente, elle se situe autour de $70 \%$. Beaucoup, donc, sont clandestins.

La concession d'une documentation légale, qui consiste en trois documents - le Registre National d'Étrangers (RNE), le Registre de personne physiques (CPF) et la Carte de Travail — garantit au réfugié l'accès à la citoyenneté brésilienne, pouvant alors bénéficier de l'ensemble de politiques sociales assurées par l'État à ses nationaux. La concession du refuge se fait par l'intermédiaire du Conseil national des réfugiés (CONARE), organisme constitué de représentants des ministères de la Justice, des Relations Extérieures, de l'Éducation, du Travail et de la Santé, ainsi que de représentants de la Police Fédérale et de Caritas Brésil, une institution sociale de l'Église Catholique. 
D'ailleurs, cette dernière se charge de la demande de refuge, de l'orientation et l'acheminement des personnes aux services, encore que provisoires, d'habitation, santé, éducation et utilisation de ressources communautaires, ainsi que des entrevues avec des représentants du CONA$\mathrm{RE}$, en raison d'un partenariat avec l'ACNUR (Haut Commissariat des Nations Unies pour les Réfugiés).

Les auberges publiques, et quelques organisations non gouvernementales d'assistance équipées de dortoirs, sont responsables de l'accueil de la grande majorité des réfugiés pendant six mois, en moyenne, ce qui oblige le réfugié à rechercher un autre logement après cette période. Mais comme il est nécessaire de séparer hommes et femmes dans les dortoirs, parents et enfants finissent par ne plus vivre ensemble.

Et s'il y a déjà des difficultés pour les brésiliens de trouver une occupation productive, le taux de chômage étant entre $10 \%$ et $18 \%$, pour l'étranger, tout sera plus difficile. Comme dans d'autres pays, la solution la plus immédiate pour la survie est, notamment, le travail au noir.

Aussi, les privations dans la vie d'un réfugié ne se résument pas simplement au plan matériel, mais sont aussi, évidemment, d'ordre psychologique et culturel. Et c'est pour pourvoir quelques unes de ces nécessités que le SESC (Service Social du Commerce) et le Senac (Service National d'Apprentissage Commercial) de São Paulo ont instauré un partenariat avec Caritas Brésil et l'ACNUR en décembre 1995, afin d'augmenter le réseau d'appui et d'offrir des conditions d'intégration à la vie sociale. Par conséquent, toute personne qui demande refuge à São Paulo est envoyée au SESC Carmo - une unité d'opérations située au centre de la ville - et là-bas, elle pourra bénéficier des services suivants:

a) cours de portugais, car un minimum de connaissance de la langue s'impose comme forme de compréhension, de socialisation et de rupture de l'isolement socioculturel ce qui mène à la reconquête de l'auto estime. Le cours a été spécialement conçu pour assouvir les inquiétudes les plus immédiates des réfugiés, ainsi que pour aborder les aspects particuliers de la vie et de la culture nationales, ce que se fait aussi par des visites aux musées et par l'assistance dans des spectacles de musique et de théâtre;

b) déjeuner et dîner au SESC Carmo, qui possède un restaurant traditionnel dans ses installations, au prix symbolique de 90 centimes de dollar américain par repas complet. Ainsi, non seulement le nouvel-arrivé s'alimente sainement, mais aussi il a l'occasion de s'adapter à la cuisine brésilienne; c) accès au réseau mondial (Internet), sous l'orientation de moniteurs, en sale spécialement conçue, dotée de structure et d'objectifs faisant partie de son programme régulier d'éducation informelle. Dans le cas des réfugiés, cette accueil journalier leur permet de rétablir ou de maintenir contact avec parents et amis ainsi que de s'informer des nouvelles de leur pays d'origine;

d) accès à des activités physiques (gymnastique) et sportives (cours et tournois) comme forme d'insertion communautaire et d'entretien individuel de la santé;

e) accès à la bibliothèque et aux espaces communs (jeux, lectures de journaux), lieux appropriés à la lecture et aux échanges d'informations avec d'autres réfugiés et habitués du centre d'activités;

f) sorties pour des spectacles artistiques gratuits et parcs, en compagnie des enfants, pour qu'ils se sentent également accueillis;

g) atelier de musique appelé «Des quatre coins du monde, " constitué d'élèves et musiciens amateurs brésiliens et réfugiés, destiné à l'apprentissage et à l'échange de genres populaires des pays d'origine des participants, dont les résultats, plus que ceux spécifiquement musicaux, se montrent dans le cadre de la convivialité et la tolérance mutuelles. Le groupe qui s'y est formé a déjà réalisé 15 présentations dans la ville, pendant les deux dernières années;

h) deux manuels d'orientation de base, en dernier lieu, pour le demandeur de refuge (2001) et le réfugié (2003) publiés par le SESC comme matériel d'appui, dans le but de les orienter au sujet de la situation, des droits, des devoirs, des normes et procédures légales du pays.

En outre, par l'intermédiaire du Senac, institution également dirigée par les entreprises du secteur tertiaire brésilien, et spécialisée dans la formation professionnelle, les réfugiés et leur famille ont l'occasion de fréquenter gratuitement les cours disponibles, du moment qu'ils possèdent les conditions requises dans chaque cas. Jusqu'à présent, à travers ce partenariat, environ 800 personnes ont suivi des cours d'informatique, photographie, administration, gastronomie, mode et santé.

Finalement, nous croyons que, même sous la précaire et douloureuse condition de réfugié, un citoyen ne peut pas être traité avec indifférence et discrimination. Le pays qui lui concède le statut de réfugié s'engage à le protéger et l'intégrer dans la société. Pour des raisons éthiques, universelles, et pour des raisons pratiques, socioéconomiques. Ainsi, la participation en coopération d'organisations gouvernementales et non gouvernementales est vitale à la survie et à la dignité de ces personnes. 


\section{SESC : culture, diversité, engagement}

Le SESC (Service Social du Commerce) est aujourd'hui, au Brésil, une référence solide et reconnue en matière de culture et d'action culturelle. Grâce aux échanges avec d'autres pays, ainsi qu'au caractère incitatif, innovateur et propositionnel des projets qu'il entreprend, son image se profile, de nos jours, bien au-delà des frontières brésiliennes.

Ce n'est pas un hasard, car il s'agit d'une institution franchement originale. En effet, on retrouve chez SESC deux aspects apparemment disparates, mais dont la conjugaison est la clef de sa singularité. C'est ainsi que, si d'une part les sources de son entretien sont assurées par la loi, d'autre part le SESC a été créé et est géré par le patronat brésilien du commerce et des services, qui le soutient avec ses contributions financières obligatoires. Le SESC est donc un organisme privé national sans but lucratif. D’une façon générale, il a l'ambition de contribuer au bien-être social, à l'amélioration de la qualité de vie et au développement culturel du travailleur du commerce et des services - qui constitue son public prioritaire — , sans exclure cependant d'autres professions ou secteurs de la société.

Dans l'État de São Paulo, le SESC dispose d'un réseau d'installations physiques constitué par 31 unités en activité actuellement. Ce sont des centres culturels et sportifs, des centres spécialisés - comme ceux de cinéma et d'odontologie - , deux centres à la campagne et un centre de vacances.

Dans ces installations, le SESC offre au public — plus d'un million de personnes par mois — des programmes et des services diversifiés, mais en même temps complémentaires, d'une amplitude d'intérêts exceptionnelle. Il s'agit concrètement de ses programmes:

a) culturels, constitués par des activités artistiques (arts plastiques, théâtre, musique, danse, littérature, vidéo et cinéma), soit sous la forme de spectacles, expositions ou festivals, soit sous celle de cours et d'ateliers. Cette rubrique comprend également les débats, les séminaires ou les congrès sur des sujets actuels ou d'importance historique;

b) de développement physique, sports et récréation, comprenant la pratique de différentes modalités sportives et des cours d'initiation divers, ainsi que la réalisation de jeux libres et récréatifs;

c) de santé - services d'odontologie et d'alimentation (restaurants et cafés), et actions éducatives, telles que des campagnes de prévention et d'information;

d) de développement psychomoteur et cognitif — destiné aux enfants de sept à douze ans, il s'effectue à travers des activités ludiques et d'information;

e) de travail social avec les personnes âgées - une action pionnière au Brésil, réunissant des groupes de troisième âge autour de Centres de Convivialité et d'Écoles Ouvertes;

f) de loisir et tourisme social — offrant des centres conçus spécialement pour les vacances et week-ends, ainsi que des voyages à plusieurs villes du Brésil;

g) d'inclusion digitale — il s'agit de salles équipées avec des ordinateurs et système d'internet, ce qui permet l'accès gratuit du public au monde digital de nos jours.

La plupart des projets ci-dessus peuvent être appuyés par des institutions publiques ou privées, brésiliennes ou étrangères, ou développés de concert avec elles. Cette possibilité de travailler en collaboration est salutaire, non seulement pour des raisons économiques, mais surtout parce qu'elle permet l'échange d'expériences, le perfectionnement des démarches, l'acquisition de nouvelles connaissances, et la perception de valeurs méconnues ou de perspectives inusitées. Cela veut dire que l'association de différentes institutions peut devenir une source d'actualisation et de réflexion continuelles, surtout aujourd'hui où les discussions s'exacerbent sur les aspects positifs et négatifs de la globalisation, sur les gains et les pertes affectant la diversité des cultures régionales ou nationales.

\section{Note}

1. S. Salgado, Exodos (São Paulo: Cia. das Letras, 2000).

Danilo Santos de Miranda est Directeur Régional du SESC da São Paulo, Brésil. 